\title{
Pour une sociologie politique de la nuit
}

Introduction

\section{CANDELA}

\section{(2) OpenEdition}

Journals

Édition électronique

URL : http://journals.openedition.org/conflits/19432

DOI : $10.4000 /$ conflits. 19432

ISSN : $1777-5345$

Éditeur :

CCLS - Centre d'études sur les conflits lilberté et sécurité, L'Harmattan

Édition imprimée

Date de publication : 15 juillet 2017

Pagination : 7-27

ISBN : 978-2-343-12713-2

ISSN : 1157-996X

Référence électronique

CANDELA, « Pour une sociologie politique de la nuit », Cultures \& Conflits [En ligne], 105-106 printemps/été 2017, mis en ligne le 15 juillet 2017, consulté le 30 mars 2021. URL : http:// journals.openedition.org/conflits/19432 ; DOI : https://doi.org/10.4000/conflits.19432 


\section{Pour une sociologie politique de la nuit 1}

Introduction

\section{CANDELA}

CANDELA est le nom d'un collectif impliquant une quinzaine de chercheur-e-s lillois-es de différents laboratoires (CERAPS, CLERSE, LATTS, TVES) : Thomas Alam, Rafaël Cos, Guillaume Courty, Antonio Delfini, Anne-Cécile Douillet, Camille Guenebeaud, David Guéranger, Nicolas Kaciaf, Paul Le Derff, Rémi Lefebore, Aurore Le Mat, Mélissa Leroy, Julien O’Miel, Aymeric Mongy, Robinson Prat, Manuel Schotté, Sidonie Verhaeghe. Le collectif s'est constitué autour du projet d'étudier comment se composent ordre public, ordre sanitaire et ordre social dans la ville nocturne. Pour une présentation de l'histoire et du fonctionnement $d u$ collectif, voir l'article paru dans ethnographiques.org : http://www.ethnographiques.org/2016/Candela.

$\mathrm{D}$ ans les représentations communes, la politique est implicitement associée au jour. Aussi les spécificités du temps social nocturne, ses appropriations et sa régulation sont-elles rarement prises en compte dans l'étude des activités politiques, du moins dans le cadre des sociétés étatiques. Comme le souligne Thomas Fouquet dans un dossier consacré aux «paysages nocturnes de la ville » en Afrique de l'Ouest, la question des temporalités est « rarement envisagée sous l'angle du rythme nycthéméral (l'alternance jour/nuit), au profit des usages sociaux et politiques du temps sur des durées plus longues 2 ». En science politique, les réflexions sur les « temporalités du politique ${ }^{3}$ » ou de l'action publique ${ }^{4}$ conçoivent le temps au regard de son écoulement et de son séquençage (construction des carrières politiques, changements en matière

1. Ce dossier dans Cultures $\mathcal{E}$ Conflits est le prolongement d'un séminaire mis en place au cours de l'année 2013-14 et d'une section thématique sur «l'ordre social nocturne » organisée lors du congrès de l'Association française de science politique à Aix-en-Provence en juin 2015.

2. Fouquet T., "Paysages nocturnes de la ville et politiques de la nuit. Perspectives ouest- africaines ", Sociétés politiques comparées, n³ 38, janvier-avril 2016, p. 2.

[http://www.fasopo.org/sites/default/files/charivaria1_n38.pdf, consulté le 11 avril 2017].

3. Pôle Sud, «Les temporalités du politique », n²5, 2006.

4. Palier B., Surel Y. et al., Quand les politiques changent. Temporalités et niveaux de l'action publique, Paris, L'Harmattan, 2010 ; Temporalités, « Temporalités et action publique », $\mathrm{n}^{\circ} 19$, 2014. 
d'action publique...), plus qu'au regard de sa structuration au quotidien. Ces travaux prêtent ainsi surtout attention aux logiques de cycles (électoraux par exemple) ou bien aux mécanismes d'imbrication entre les rythmes propres à la compétition politique, aux activités médiatiques et à la fabrique de l'action publique, cette dernière pouvant être prise dans des logiques d'urgence ou, au contraire, de planification. Quant aux recherches récentes sur les emplois du temps d'élus ${ }^{5}$ ou sur le rapport au temps (genré) des hauts fonctionnaires ${ }^{6}$, elles examinent effectivement les usages sociaux du temps quotidien mais n'accordent pas d'attention particulière à la nuit, insistant plutôt sur la saturation des agendas des édiles locaux ou sur les arbitrages, socialement différenciés, entre «consommation ostentatoire » et « gestion bourgeoise » du temps ${ }^{7}$.

Cet impensé relatif explique sans doute l'étonnement des initiateurs de CANDELA lorsque, au milieu d'une nuit de juillet 2012, ils croisent la marcha negra des mineurs des Asturies alors qu'ils se trouvent à Madrid à l'occasion d'un congrès international de science politique. Les mineurs protestent contre la fermeture des mines et leur arrivée dans la capitale espagnole donne lieu à un grand rassemblement ${ }^{8}$. Si l'intrusion d'un mouvement social et l'expression de revendications dans la nuit ont pu surprendre, c'est que la politique est arrivée quand on ne l'attendait pas vraiment, l'heure étant plutôt au sommeil, aux sorties festives ou à l'intimité domestique 9.

Pourtant, l'activité politique est loin d'être étrangère à la nuit. Celle-ci peut ainsi être l'occasion d'événements politiques, qu'ils soient exceptionnels ou qu'ils relèvent de pratiques routinisées. La célèbre « Nuit du 4 août » de la Révolution française ${ }^{10}$, de même que les barricades du printemps des peuples 11 ou de mai 196812 témoignent de l'existence de «nuits historiques ».

5. Lefebvre R., "Les élus comme entrepreneurs de temps. Les agendas des cumulants », in Demazière D., Le Lidec P. (dir.), Les mondes du travail politique. Les élus et leurs entourages, Rennes, PUR, 2014, pp. 53-70 ; Godmer L., Marrel G., La politique au quotidien. L'agenda et l'emploi du temps d'une femme politique, Lyon, ENS Éditions, 2016.

6. Favier E., «Pourquoi une présence au bureau de quinze heures par jour ? Rapports au temps et genre dans la haute fonction publique », Revue française d'administration publique, $\mathrm{n}^{\circ} 153$, 2015.

7. Ibid., p. 89.

8. Voir sur le site du quotidien El Pais : http://ccaa.elpais.com/ccaa/2012/07/10/madrid/ 1341904617_371442.html [consulté le 11 avril 2017].

9. La nuit est aussi un temps dévolu au travail pour un nombre croissant de salariés. Une étude de la DARES de 2012 indique que « 15,4\% des salariés $(21,5 \%$ des hommes et 9,3\% des femmes), soit 3,5 millions de personnes, travaillent la nuit, habituellement ou occasionnellement ", soit un million de salariés de plus qu'en 1991 [http://travailemploi.gouv.fr/IMG/pdf/2014-062.pdf, consulté le 11 avril 2017].

10. Bourdin P. (dir.), Les nuits de la Révolution Française, Clermont-Ferrand, Presses Universitaires Blaise Pascal, 2013 ; Tackett T., Par la volonté du peuple. Comment les députés de 1789 sont devenus révolutionnaires, Paris, Albin Michel, 1997.

11. Voir par exemple Engels F., «Le 24 juin ou les journées de juin 1848 », Nene Rheinische Zeitung, 28 juin $1848, \mathrm{n}^{\circ} 28$, p. 2.

12. Voir, par exemple, sur la nuit du vendredi 10 mai 1968 : http://www.ina.fr/video/CAF89036278 [consulté le 11 avril 2017]. 
Qu'elles fassent date ou non, les grandes négociations sociales et internationales se prolongent souvent tard et la capacité « à tenir la nuit » y est importante. Dans un registre plus institutionnel et conventionnel, les sessions parlementaires peuvent empiéter sur la nuit, par exemple lors des interminables débats sur la loi de finances.

La nuit semble même avoir été investie d'une dimension politique renouvelée ces dernières années. Ainsi, la mobilisation des « veilleurs » de la « Manif pour tous » en 2013 ou les manifestations nocturnes des policiers français en octobre 2016 soulignent combien l'affichage d'une capacité à rester mobilisé, y compris la nuit, fait partie du répertoire de l'action collective contemporaine. Dans un cadre très différent, le mouvement « Nuit Debout » du printemps 2016 - après le $15 \mathrm{M}$ espagnol, le mouvement Occupy et la longue occupation de la place Tahrir au Caire - démontre très directement, jusque dans sa dénomination, en quoi l'appropriation citoyenne de l'espace public nocturne participe de l'invention de nouvelles manières de faire de la politique. La mobilisation politique nocturne est ici défendue comme permettant un brassage social significatif, par opposition à la « journée de travail » qui tend à segmenter et hiérarchiser les groupes sociaux. Les enquêtes menées lors du mouvement « Nuit Debout » n'en révèlent pas moins des variations dans le profil des participants selon les heures, rappelant notamment l'importance des disponibilités biographiques et familiales, aussi saillantes la nuit que le jour ${ }^{13}$.

La nuit n'est pas seulement un espace-temps de mobilisation, elle peut aussi être un enjeu de mobilisation. C'est ce qu'illustrent par exemple les manifestations féministes Take back the night qui se sont développées, d'abord en Amérique du Nord, à partir des années 1970 : dénonçant les formes de domination vécues par les femmes dans l'espace public nocturne, elles visent à une réappropriation de la nuit. L'organisation de marches nocturnes est alors en lien direct avec l'objet de la mobilisation.

Enfin, la nuit est un objet d'action publique relativement institutionnalisé, à l'échelle municipale ou métropolitaine en particulier. En témoignent ces bureaux des temps qui fleurissent depuis les années 2000 dans l'espoir de mieux concilier horaires de travail, transports et services, notamment la nuit ${ }^{14}$. Les " politiques de la nuit » apparaissent en tension entre impératifs

13. Plusieurs recherches ont été engagées pour caractériser sociologiquement le mouvement « Nuit Debout». Tandis que les données collectées par Geoffrey Pleyers donnent à voir un groupe relativement jeune, majoritairement masculin, diplômé du supérieur et marqué par la précarité [https://www.politis.fr/articles/2016/05/qui-sont-les-participants-de-nuit-debout34685, consulté le 11 avril 2017], l'enquête de Stéphane Baciocchi, Alexandra Bidet et al. confirme ces observations générales mais souligne la variabilité de la morphologie sociale du groupe selon les heures du jour et de la nuit [http://www.lemonde.fr/idees/ article/2016/05/17/nuit-debout-est-un-rassemblement-plus-diversifie-qu-on-ne-ledit_4920514_3232.htm, consulté le 11 avril 2017].

14. Mallet S., "Des politiques temporelles à un urbanisme temporel ? », in Vassallo P., Royoux 
d'ordre public ${ }^{15}$ et logiques de marketing territorial ${ }^{16}$, dans un contexte de développement des activités récréatives nocturnes. Espaces festifs et autres entertainment zones participent de la construction plus ou moins planifiée d'urban nightscapes 17 intégrés aux projets de régénération urbaine qui remodèlent les centres urbains post-industriels ou post-fordistes ${ }^{18}$. Les collectivités territoriales voient dans le développement de ce secteur économique un moyen de renforcer l'attractivité des villes auprès des touristes, des investisseurs, des «classes créatives 19 » et autres «city breakers », et de créer ainsi de la richesse et de l'emploi 20.

Si la nuit demeure un objet encore peu exploré par la science politique, elle n'est pas une terra incognita pour les sciences sociales. Les travaux anthropologiques interrogent depuis longtemps la « nocturnité » 21 , soulignant notamment que la nuit « sociale » ne peut se définir uniquement par l'absence de lumière 22 ou être réduite à la « nuit solaire ». Elle renvoie à un espace-temps

D. (dir.), Urgences temporelles. L'action publique face au temps de vivre, Paris, Éditions Syllepse, 2013.

15. Voir par exemple le projet «Safer drinking scenes 2011-13 : Les jeunes et la consommation excessive d'alcool dans l'espace public » piloté par le European Forum for Urban Security [https://efus.eu/fr/topics/risks-forms-of-crime/substance-abuse/efus/1983/, consulté le 11 avril 2017].

16. Sur ces tensions et les arbitrages qu'elles engendrent, voir les cas contrastés et mouvants de Rennes (dans ce numéro), de Londres depuis l'élection de Sadiq Khan [http://www.bbc.com/news/uk-england-london-36332868], de Sydney [https://www. theguardian.com/australia-news/2016/feb/05/sydneys-fun-police-have-put-out-the-lightof-the-nightlife-the-citys-a-global-laughing-stock] ou encore de Tokyo [http://www. telegraph.co.uk/travel/destinations/asia/japan/articles/Japan-may-ease-ban-on-dancing/]. Pages consultées le 11 avril 2017.

17. Voir Lovatt A., O'Connor J., "Cities and the Night-time Economy », Planning Practice E Research, vol. 10, n² 2, 1995, pp. 127-134 ; Chatterton P., Hollands R., Urban Nightscapes: Youth Cultures, Pleasure Spaces and Corporate Power, London, Routledge, 2003 ; Roberts M. et Eldridge A., Planning the Nighttime City, Abingdon, Routledge, 2009. Antoine De Baeque montre pour sa part l'attractivité précoce de Paris pour ses nuits (Les nuits parisiennes. XVIII $-X X I^{e}$ siècle, Paris, Le Seuil, 2015).

18. Harvey D., «From Managerialism to Entrepreneurialism: the Transformation of Urban Governance in Late Capitalism ", Geografiska Annaler, Series B, Human Geography, vol. 71, $\mathrm{n}^{\circ} 1,1989$, pp. 3-17.

19. Florida R., The Rise of the Creative Class. And How It's Transforming Work, Leisure and Everyday Life, New York, Basic Books, 2002.

20. Cette stratégie marketing ne s'élabore pas seulement à l'échelle municipale. Ainsi le «tourisme nocturne » a-t-il été identifié en 2015 par le ministère des Affaires étrangères comme l'un des cinq pôles d'excellence permettant d'améliorer l'attractivité et le rayonnement de la France [http://www.diplomatie.gouv.fr/IMG/pdf/feuille_de_route_pole_nuit_finalisee_ cle4add54-1.pdf]. Sur les affinités entre city-breakers et vie nocturne, voir Guérin F., "L'easyjet-setting de Paris à Berlin ", Téoros, vol. 34, $\mathrm{n}^{\circ}$ 1-2, 2015 [https://teoros.revues.org/2731, consulté le 11 avril 2017].

21. Pour une synthèse des travaux d'anthropologues sur la nuit, voir Galinier J., Monod Becquelin A. et al., "Anthropology of the Night. Cross-Disciplinary Investigations ", Current Anthropology, vol. 51, n 6, 2010, pp. 819-847. Voir aussi les réflexions portées par le séminaire "Anthropologie de la nuit » du LESC (Laboratoire d'Ethnologie et de Sociologie comparées), organisé depuis plusieurs années à l'université de Paris-Nanterre.

22. La recherche de Guy Bordin (On dansait seulement la nuit. Fêtes chez les Inuits du nord de la Terre de Baffin, Nanterre, Publications de la Société d'ethnologie, 2012) sur les activités des Inuits du Nunavut, une zone géographique avec des nycthémères très particuliers (les jours et 
de pratiques, d'attitudes, et de comportements sociaux singuliers dans toutes les sociétés (quelles qu'en soient les délimitations, la nuit se distingue du jour), mais dont les modalités s'avèrent particulièrement différenciées selon les contextes sociohistoriques. Le statut des activités oniriques nocturnes en est une illustration : tandis que les rêves se conçoivent chez nous comme l'une des pratiques les plus intimes, Anne-Gaëlle Bilhaut montre à quel point ils sont centraux dans la vie collective des Indiens Zapara car ils fournissent un réservoir de symboles orientant l'action de la communauté ${ }^{23}$. D'autres auteurs font de la nuit le temps privilégié des pratiques occultes et magiques, qu'il s'agisse des sorciers de Douala ${ }^{24}$, des conseillers des dirigeants indonésiens ${ }^{25}$, des chamanes Otomi ${ }^{26}$ ou encore de l'anthropophagie symbolique des sorciers camerounais à des fins économiques ${ }^{27}$, pour ne citer que quelques exemples.

Tout en invitant à déconstruire nos prénotions relatives aux « heures sombres » et à leur appréhension par les pouvoirs politiques, les recherches en histoire ont pour leur part mis en évidence que la dimension politique de la nuit ne constituait pas une originalité de l'époque contemporaine. C'est le cas en particulier dans les travaux de Carlo Ginzburg ou Jean Verdon sur la nuit médiévale : ils y montrent à quel point l'obscurité joue un rôle symbolique important comme métaphore de l'obscurantisme païen (la déviance, la monstruosité, le diabolique) ${ }^{28}$. Travaillant sur des périodes plus récentes (XVII ${ }^{\mathrm{e}}$-XIX ${ }^{\mathrm{e}}$ siècle), plusieurs historiens ${ }^{29}$ ont récemment rappelé que la «colonisation » de la nuit par l'éclairage public est fondamentalement politique tant il permet de mieux contrôler l'espace nocturne, tout en faisant l'objet de résistances en raison notamment des nouveaux impôts qui y sont liés 30 .

nuits polaires sans obscurité ou sans lumière pendant plus de 24 heures), met clairement à distance le lien entre nuit et absence de lumière et montre comment le temps peut être mis sens dessus dessous : chez les Inuits, la nuit d'été sans obscurité est valorisée, notamment pour la chasse et la pêche, alors que, lors des hivers obscurs, on fait la fête la nuit jusqu'au petit matin et on dort pendant la journée sombre.

23. Bilhaut A.-G., Des nuits et des rêves. Construire le monde Zápara en Haute Amazonie, Nanterre, Société d'ethnologie, 2011.

24. De Rosny E., Les yeux de ma chèvre. Sur les pas des maîtres de la nuit en pays donala (Cameroun), Paris, Plon, 1981.

25. Bertrand R., Indonésie : la démocratie invisible. Violence, magie et politique à Java, Paris, Karthala, 2002.

26. Galinier J., Une nuit d'épouvante. Les indiens Otomi dans l'obscurité, Nanterre, Publication de la Société d'ethnologie, 2011.

27. Geschiere P., Sorcellerie et politique en Afrique. La viande des autres, Paris, Karthala, 1995. Il y est notamment question de la « sorcellerie de la richesse » et de l'exploitation nocturne des « zombies».

28. Ginzburg C., Les batailles nocturnes. Sorcellerie et rituels agraires an XVI et XVII siècle, Paris, Champs Flammarion, 1984 ; Verdon J., La nuit au Moyen Âge, Paris, Perrin, 1994.

29. Schlor J., Nights in the Big City: Paris, Berlin, London; 1840-1930, London, Reaktion Books, 1998 ; Ekirch A. R., At Day's Close: A History of Night-time, London, Weidenfeld and Nicholson, 2005 ; Delattre S., Les donze heures noires : la nuit à Paris au XIXe siècle, Paris, Albin Michel, 2000 ; Cabantous A., Histoire de la nuit (xviie-xviiie siècles), Paris, Fayard, 2009 ; De Baecque A. de, Les nuits parisiennes, op. cit.

30. C'est à la lanterne, associée au pouvoir, que les révolutionnaires pendent les aristocrates. 
Il ne fait donc aucun doute que la nuit, son appréhension et ses appropriations, comportent de très nombreuses dimensions politiques. Au-delà de l'imaginaire associé aux événements qui s'y déroulent, elle constitue un enjeu d'affrontements politiques, tant elle conditionne, en même temps qu'elle révèle, certains rapports sociaux (de classe, de genre, de racialisation, etc.). Espace-temps aux frontières indéterminées et mouvantes, la nuit et ses usages sont par conséquent l'objet d'investissements politiques menés par différents groupes ainsi que par les autorités chargées d'arbitrer entre leurs prétentions et de réguler sinon domestiquer la vie nocturne.

Ce sont ces différentes dimensions politiques de la nuit que le collectif CANDELA cherche à explorer. Ce numéro thématique participe de cette entreprise de sociologie politique de la nuit, en ouvrant la réflexion sur divers contextes politiques et sociaux (France, Russie, Sénégal, États-Unis...). Il est notamment l'occasion de soulever une interrogation que cette introduction entend clarifier : quel peut être le statut de l'objet « nuit » dans les sciences sociales du politique ? Faut-il appréhender la nuit comme une séquence temporelle objectivement porteuse de pratiques et de rapports sociaux originaux au regard de ce que les sciences sociales observent le jour, ou bien avant tout comme une catégorie aux définitions fluctuantes mais servant de cadre à la construction de problèmes publics et à l'intervention des pouvoirs publics ? On peut par ailleurs se demander si les caractéristiques de la vie nocturne constituent un simple redoublement de l'ordre social et politique diurne, ou si elles donnent à voir des phénomènes spécifiques et potentiellement subversifs. La lecture croisée des articles réunis dans ce numéro permet de répondre à ces questions en faisant émerger trois appréhensions de la nuit et de sa démarcation avec le jour.

\section{Quand la nuit « révèle » et prolonge le jour}

Une première hypothèse sous-jacente au projet CANDELA était que l'analyse de ce qui se joue lors des « heures sombres » permet de mettre au jour certaines caractéristiques de l'ordre social et politique des sociétés contemporaines, invisibles ou moins facilement saisissables le jour. En étudiant la nuit, et en particulier la nuit urbaine, le géographe, le politiste ou le sociologue retrouvent en effet des objets et des questionnements familiers : les conflits d'usage de l'espace, l'expression des rapports sociaux dans la sphère publique, le développement et l'institutionnalisation de nouveaux métiers, la centralité des enjeux de sécurité et de développement (commerciaux ou touristiques) dans les politiques locales, les concurrences intra- ou interprofessionnelles...

L'intérêt d'une entrée par la nuit serait alors de montrer dans quelle mesure certaines de ces logiques sociales et politiques y sont exacerbées. Dans le cas des concierges des hôtels de luxe étudiés par Thibaut Menoux, le travail 
de nuit met ainsi en évidence de manière particulièrement criante le caractère subalterne du métier dans la relation aux clients (même si l'extravagance de certaines demandes à ces heures rend aussi possibles des formes originales de convivialité). L'étude du travail de nuit permet aussi de préciser les hiérarchies qui structurent le groupe professionnel (en fonction des origines, du capital scolaire ou du parcours professionnel), qui se réfractent ici dans les clivages entre concierges de jour et concierges de nuit. «Lieu » de recherche autant qu'objet de recherche qui se suffirait à lui-même ${ }^{31}$, la nuit peut ainsi être envisagée à la fois comme une loupe, un « révélateur » (au sens photographique du terme) ${ }^{32}$, ou encore un « levier de connaissance » pour emprunter l'expression proposée par Thibaut Menoux dans ce numéro.

Dans certaines analyses, la nuit apparaît, plus que comme un simple prolongement $\mathrm{du}$ jour, comme un territoire conquis pour y imposer l'ordre politique et économique qui gouverne le jour. Dès 1867, Karl Marx analysait le travail de nuit - forme ultime d'exploitation de la force de travail - comme le symptôme de cette gloutonnerie du capital qui dépasse «non seulement les limites morales, mais encore la limite physiologique extrême de la journée de travail 33 ». Pour Jonathan Crary, «Open 24/7 » serait devenu le mot d'ordre du capitalisme contemporain, un capitalisme à l'assaut du sommeil, grignotant sans cesse le temps consacré au repos et au rêve, dans sa logique d'expansion continue ${ }^{34}$. Aux activités de production industrielle et aux services d'urgence 35 s'ajoutent en effet les pratiques de consommation, génératrices d'une nouvelle « économie de nuit ». Cette Night-time Economy ${ }^{36}$ s'inscrit dans le sillage de l'économie du jour et en durcit les logiques : concentration capitalistique, financiarisation, branding, aseptisation, segmentation des marchés, jusqu'aux processus de spéculation immobilière et d'exclusion de certaines catégories d'habitants, surtout quand les municipalités participent elles-

31. Un rapprochement peut ici être fait avec l'approche localisée du politique (Briquet J.-L., Sawicki F., "L'analyse localisée du politique. Lieux de recherche ou recherche de lieux », Politix, n 7, 1989, pp. 6-16). Vincent Dubois souligne ainsi que « recourir à l'analyse localisée ce n'est de fait pas forcément étudier le local pour lui-même, ni comme simple réceptacle d'orientations définies ailleurs. Ce peut être en revanche utiliser un cadre territorial comme base d'observation. » («Éléments pour une socio-histoire des politiques culturelles locales. Enjeux et perspectives d'une analyse localisée », in Dubois V. (dir.), Le politique, l'artiste et le gestionnaire. (Re)configurations locales et (dé)politisation de la culture, Bellecombe-enBauges, Le Croquant, 2012, p. 207). Cette citation fait en quelque sorte écho au propos de Thibaut Menoux dans ce numéro lorsqu'il explique qu'il s'agit de dépasser à la fois la « variation temporelle sur un même thème » et l'analyse d'un «monde à part ». Dans l'analyse localisée du politique, la dialectique entre attention aux particularités et montée en généralité est du même ordre que celle qui est défendue ici dans l'étude du politique la nuit.

32. En photographie argentique, le révélateur assombrit les parties exposées à la lumière, les révélant ainsi à l'œil. De même, la nuit permet de voir, par contraste, ce qu'on ne voit plus le jour.

33. Marx K., Le Capital. Livre I (1867), Paris, PUF, 2009, p. 293.

34. Crary J., 24/7. Le capitalisme à l'assaut du sommeil, Paris, La Découverte, 2014.

35. Sharman R.L., Sharman C.H., Nightshift NYC, Berkeley, University of California Press, 2008.

36. Lovatt A., O’Connor J., "Cities and the Night-time Economy », Planning Practice $\varepsilon$ Research, op. cit. ; Hadfield P., Bar Wars. Contesting the Night in Contemporary British Cities, Oxford, Oxford University Press, 2006, 
mêmes à une sectorisation géographique et oligopolistique des activités récréatives nocturnes ${ }^{37}$. Au Royaume-Uni, le temps où le pub était l'institution centrale de la communauté pour la working class des cités industrielles est bel et bien révolu ${ }^{38}$. Non seulement le nombre de "public houses » est en diminution significative (99 500 en 1904, 69000 en 1980 et 51000 en 2010), mais leur business model est radicalement transformé et concurrencé par les bars dansants, les night-clubs mainstream des high streets 39 et, dans une moindre mesure, par les espaces alternatifs indépendants et plus distingués aux marges du centre-ville. Au croisement de ces stratégies commerciales, immobilières et de peuplement, la vie nocturne participe à l'embourgeoisement de quartiers anciens, post-industriels et populaires, comme le montrent les études sur la rue Oberkampf à Paris ${ }^{40}$, sur la place Saint-Géry et la rue Antoine Dansaert à Bruxelles ${ }^{41}$, sur la gentrification de Downtown Manhattan, Harlem ou Brooklyn à New York ${ }^{42}$, ou sur celle des quartiers de Gemmayzeh, de Mar Mikhail et de Hamra à Beyrouth 43. Dans le district gentrifié de Maboneng, au sein du quartier populaire de Jeppestown à Johannesburg, les rooftops symbolisent toute l'ambiguïté des rapports aux espaces nocturnes des « gentrifieurs » : «l'espace juridiquement privé du roof-

37. À Lille, bars, clubs et kebabs se sont d'abord progressivement implantés dans les espaces laissés vacants par la fermeture des Halles centrales en 1976, avant de bénéficier d'avantages spécifiques attribués par la ville (autorisations de fermeture tardives jusqu'à $3 \mathrm{~h}$ du matin réservées aux seuls bars de deux secteurs situés en plein cœur de ville). Voir CANDELA, «Concentrer la vie nocturne pour mieux la contrôler ? La sectorisation à Lille, entre volontarisme politique et logiques marchandes » (in Guérin F. et al., Cohabiter les nuits urbaines, Rennes, Presses Universitaires de Rennes, à paraitre en 2017) et l'encadré ${ }^{\circ} 3$ sur le public de ces bars.

38. Mass Observation, The Pub and the People. A Worktown Study, Welwyn Garden City Seven Dials Press, 1970.

39. En 1989, 88 \% des pubs étaient gérés par des brasseurs ou reliés à eux par un système de gérance. Au début des années 2000, les «pubcos» (ou public estate companies cotées en bourse) détiennent désormais la moitié des pubs, d'où le caractère standardisé des quartiers nocturnes britanniques. Il en est de même pour les clubs, qui ont pourtant fait la notoriété de certaines villes (comme la mythique Hacienda à Manchester). Ils sont désormais administrés par de nouveaux géants de la nuit comme le PoNaNa Group ou Luminar Leisure (15\% des clubs pour ce dernier au début des années 2000). Voir Chatterton P., Hollands R., «Producing Nightlife in the New Urban Entertainment Economy: Corporatization, Branding and Market Segmentation ", International Journal of Urban and Regional Research, vol. 27, n 2, 2003, pp. 361-385 et Roberts M., Townshend T., "Young adults and the decline of the urban English pub: issues for planning ", Planning Theory E Practice, vol. $14, n^{\circ} 4,2013$, pp. 455-469.

40. Fleury A., "De la rue-faubourg à la rue "branchée" : Oberkampf ou l'émergence d'une centralité des loisirs à Paris », L’Espace géographique, vol. 32, n 3, 2003, pp. 239-252.

41. Van Criekingen M. et Fleury A., « La ville branchée : gentrification et dynamiques commerciales à Bruxelles et à Paris», Belgeo, n ${ }^{\circ}$-2, 2006, pp. 113-134.

42. Zukin S., "Où sont passés les cafés du coin ? ", Ethnologie française, vol. 36, n 4, 2006, pp. 749-752 ; Zukin S., Naked City. The Death and Life of Authentic Urban Places, Oxford, Oxford University Press, 2010 ; Hae L., "Gentrification and Politicization of Nightlife in New York City ", ACME: An International E-Journal for Critical Geographies, vol. 11, $\mathrm{n}^{\circ} 3$, 2011, pp. 564-584 ; Ocejo R., Upscaling Downtown: From Bowery Saloons to Cocktail Bars in New York City, Princeton, Princeton University Press, 2014.

43. Bonte M., Le Douarin L., « Dans les pas de la nuit. Les rythmes urbains de Beyrouth à la tombée du jour ", Revne des mondes musulmans et de la Méditerranée, n 136, novembre 2014 [http://remmm.revues.org/8899, consulté le 11 avril 2017]. 
top tend à restreindre l'accessibilité sociale du lieu et permet la domination symbolique, par le regard, d'un centre-ville nocturne qui, sous d'autres modalités, inspire plutôt la peur ${ }^{44}$ ».

S’intéressant aux rapports sociaux de domination, certains auteurs mettent pour leur part en évidence une asymétrie dans le « droit à la ville 45 » particulièrement sensible la nuit. La distribution spatiale et le contenu des sorties nocturnes sont gouvernés par l'imbrication des rapports sociaux de sexe, de sexualités, de classe, de racialisation et d'âge ${ }^{46}$. Comme le souligne le travail réalisé par Camille Guenebeaud, Aurore Le Mat et Sidonie Verhaeghe (collectif CANDELA) sur les rapports sociaux de sexe dans l'espace public nocturne à Lille, il n'y a rien là de spécifique à la nuit mais les rapports de domination sont plus fortement ressentis la nuit (voir encadré $n^{\circ} 1$ ). C’est aussi ce qui ressort de l'article de Patrick Bruneteaux dans ce numéro : il montre notamment que les femmes SDF, surtout quand elles sont seules, sont, plus que les hommes, condamnées à rester éveillées la nuit ("à proximité de l'affluence et de la luminosité »), du fait d'un sentiment de grande vulnérabilité et d'une crainte de l'agression. Elles sont ainsi en quelque sorte privées de nuit.

\section{Encadré 1 : La rue, la nuit, femmes sans peur ?}

En août 2012, une rubrique «Conseils aux femmes » publiée sur le site internet du ministère de I'Intérieur adresse plusieurs recommandations aux femmes qui s'aventurent dans les espaces publics : «éviter les lieux déserts, les voies mal éclairées, les endroits sombres où un éventuel agresseur peut se dissimuler»; "Dans la rue, marchez d'un pas énergique et assuré, ne donnez pas l'impression d'avoir peur ». Ces « conseils » sont symptomatiques des imaginaires sociaux et culturels qui entourent la rue la nuit et des représentations sexistes sur la différence et la fragilité des femmes.

44. Oloukoï C., « Nuits, objets de peurs et de désirs à Maboneng (Johannesburg, Afrique du Sud) ", Sociétés politiques comparées, $\mathrm{n}^{\circ} 38$, janvier-avril 2016, p. 6 [http://www.fasopo.org/sites/default/files/charivaria2_n38.pdf, consulté le 11 avril 2017$].$

45. Lefebvre H., Le droit à la ville (1968), Paris, Economica, 2009.

46. Koskela H., Fear, Control and Space: Geographies of Gender, Fear of Violence, and Video Surveillance, Helsinki, Publications of the Department of Geography, 1999 ; Réau B., "Enchantements nocturnes : ethnographie de deux discothèques parisiennes », Ethnologie française, vol. 36, n² 2, 2006, pp. 333-339; Talbot D., Regulating the Night. Race, Culture and Exclusion in the Making of the Night-time Economy, London, Routledge, 2007 ; Salomon C., "Jungle Fever. Genre, âge, race et classe dans une discothèque parisienne ", Genèses, n 69, 2007, pp. 92-111; Bacqué M.-H., Fol S. «L'inégalité face à la mobilité : du constat à l'injonction ", Revue suisse de sociologie, vol. 33, n¹, 2007, pp. 89-104 ; Lieber M., Genre, violences et espaces publics. La vulnérabilité des femmes en question, Paris, Presses de Sciences Po, 2008 ; Allemand R., «De la mystification des pratiques à la négation du réel ? Ethnographie de discothèques montpelliéraines ", Déviance et Société, vol. 34, $\mathrm{n}^{\circ} 1,2010$, pp. 29-48 ; Burnett J., "UK: racial violence and the night-time economy ", Race E Class, vol. 53, n 1, 2011, pp. 100-106 ; Fouquet T., "Aventurières noctambules », Genre, sexualité Esociété, n 5, printemps 2011 [https://gss.revues.org/1922] ; Bonte M., "Gay Paradise Kind of”. Les espaces de l'homosexualité masculine à Beyrouth », EchoGéo, n² 25, juillet-septembre 2013 [https://echogeo.revues.org/13498]. 
Trois membres de CANDELA ont mené une recherche sur le sexisme dans I'espace public nocturne lillois, construite autour d'entretiens avec une vingtaine de femmes ${ }^{47}$, de la réalisation de cartes mentales, d'observations nocturnes et de comptages dans l'espace public ${ }^{48}$. Les expériences recueillies montrent que l'inattention civile, l'indifférence et l'anonymat, qui constituent pour Goffman les normes qui régissent les relations sociales dans les villes ${ }^{49}$, ne sont pas respectées pour les femmes. Elles sont en effet fréquemment sujettes à des interactions non désirées avec des inconnus dans l'espace public. Cette communauté d'expériences des femmes n'a cependant pas de spécificité nocturne. D'après les données de l'enquête ENVEFF (Enquête nationale sur les violences envers les femmes en France, 2000), les agressions, quel qu'en soit le type, ne sont pas nécessairement produites à la faveur de l'obscurité puisque la majorité d'entre elles $(67,2$ \%) ont lieu dans la journée ou tôt le matin.

La nuit ne changerait donc rien pour les femmes? Ce n'est pas ce qui ressort des entretiens : pour les femmes interrogées, les déplacements nocturnes s'accompagnent de changement dans leurs ressentis et dans leurs pratiques de l'espace urbain. Les trajets peuvent par exemple être modifiés de nuit, afin d'éviter des rues peu fréquentées. "Je vais être un peu stratégique quand même. La nuit, je préfère prendre les grands axes ", explique Mathilde, 25 ans. Les modes de déplacement sont anticipés : il peut s'agir de prendre son vélo ou sa voiture pour éviter de rentrer seule à pied ou en métro. "En vélo, t'évites les regards, tu vas vite, t'es pas dans la même dimension ", suggère Estelle, 22 ans. Celles qui se déplacent à pied utilisent parfois des objets pour signifier leur indisponibilité, comme Pauline, 22 ans, qui passe systématiquement des appels téléphoniques quand elle est seule dans la rue la nuit afin de «ne pas être obligée de rétorquer si on [l]'interpelle».

Ces changements ne sont pas liés à des interactions ou à des agressions qui seraient spécifiques à la nuit, mais sont plutôt les conséquences des représentations qui lui sont attachées, à l'instar de ces « Conseils aux femmes » du ministère de l'Intérieur. II ne s'agit pas de dire que les peurs de certaines femmes sont infondées, mais que la répétition et l'omniprésence des discours sur la rue la nuit contribuent à imposer une perception de ce que doit être la place des femmes. Malgré l'absence d'interdiction pure et simple de I'accès des femmes aux espaces publics la nuit, l'enquête montre qu'il existe une circonscription des espaces dans lesquels elles peuvent légitimement être et se déplacer. En pensant un espace (la rue) et un temps (la nuit) comme criminogènes par essence, plutôt que $d^{\prime}$ 'interroger le rapport de pouvoir et de domination qui s'exerce par les hommes sur les femmes dans

47. Malgré une attention portée à la diversité des profils des personnes interviewées en termes de classe socio-économique, d'âge, de racialisation, de sexualité et de situation familiale, les entretiens effectués ont été réalisés en majorité avec des personnes entre 20 et 35 ans, blanches et de classe moyenne.

48. Les résultats de cette enquête ont été présentés lors de plusieurs communications orales, notamment lors de la journée d'études "Sortir la nuit. Rapports sociaux de sexe, perceptions et usages nocturnes des espaces publics » organisée le 26 novembre 2013 à l'Université Lille 2, et lors du colloque "Cohabiter les nuits urbaines ", organisé par l'Université Paris Est à Marne-la-Vallée, le 5 mars 2015.

49. Voir Goffman E., La Mise en scène de la vie quotidienne. Vol. 2 : Les relations en public, Paris, Éditions de Minuit, 1973 et Comment se conduire dans les lieux publics. Notes sur l'organisation sociale des rassemblements, Paris, Economica, 2013. 
les espaces publics la nuit ${ }^{50}$, cela renvoie les femmes à certaines places (les foyers, les familles), là où elles sont censées être en sécurité. C'est pourtant dans l'espace privé constitué du couple, de la famille et des proches, que se produisent la majorité des violences physiques et sexuelles envers les femmes (ENVEFF, 2000). En mettant en avant le risque que les femmes prennent à sortir dans la rue le soir ou la nuit, ces discours mobilisent la peur comme moyen de contrôle sur leurs vies, leurs déplacements et leurs sorties.

Tous ces travaux mettent en évidence que les logiques économiques, sociales et politiques qui structurent le fonctionnement social ne changent pas fondamentalement la nuit. La focalisation sur celle-ci se justifie alors par le fait qu'elle les révèle parfois de façon plus nette et plus criante (le plus grand sentiment de vulnérabilité des femmes dans l'espace public nocturne en témoigne). Pour analyser cette importation nocturne des principes constitutifs de la vie diurne, Murray Melbin - dont nous traduisons dans ce numéro le texte paru en 1978 dans l'American Sociological Review, "Night as Frontier »-opère une comparaison avec le processus de conquête territoriale dans l'Ouest américain du XIXe siècle. Il y décrit une nuit initialement peuplée de quelques pionniers qui l'entrevoient comme un nouvel espace des possibles, suspendant au moins partiellement certaines des logiques du jour, en favorisant par exemple des relations plus altruistes ${ }^{51}$. Mais s'il montre que la nuit change les relations sociales, de la même façon que la vie dans les villes et villages créés par les pionniers s'organisait différemment de celle sur la côte Est, sa comparaison avec la conquête de l'Ouest sous-entend une forme de «normalisation », les différenciations entre l'Ouest et l'Est s'étant considérablement estompées au fil du temps.

\section{Quand la nuit appelle des régulations spécifiques}

Faire une "sociologie nyctalope " (Thibaut Menoux, dans ce numéro) dans la logique qui vient d'être exposée, n'interdit pas de questionner la façon dont l'espace-temps de la nuit est spécifiquement investi, socialement et politiquement. C'est même précisément en regardant de près comment l'alternance (naturelle) nuit/jour est appropriée et vécue par une pluralité de groupes sociaux, que l'on peut faire de la nuit un analyseur de la structuration sociale. Avant de penser la nuit comme un « levier de connaissance », le collectif CANDELA l'avait d'ailleurs initialement envisagée comme une

50. Il est entendu qu'il n'est aucunement question de réduire cette domination au seul espacetemps dont il est ici question.

51. Stimulante par ses intuitions et son imagination sociologique, la démonstration de Melbin procède par un usage métaphorique de la comparaison avec la conquête de l'Ouest plus que par une analyse serrée de la congruence des processus à l'œuvre. Par ailleurs, s'appuyant principalement sur Turner, l'un des historiens « officiels» de l'extension territoriale des EtatsUnis, Melbin donne à voir la conquête de l'Ouest - étonnement pacifique - comme celle d'une terre inhabitée. 
séquence temporelle «à part ». C'est une telle perspective qui a nourri le projet de mise au jour des régulations nocturnes. L'hypothèse était que la nuit - en tant que séquence « extra-ordinaire » du point de vue aussi bien des catégories de l'entendement social que de celles de l'action publique 52 - devait donner lieu à des interventions spécifiques, le temps « ordinaire » de la vie sociale étant supposé être le jour. CANDELA a ainsi initié différentes enquêtes sur le terrain lillois, pour appréhender à la fois les régulations sociospatiales (via l'analyse des rapports sociaux de sexe dans les espaces publics nocturnes ${ }^{53}$ ou l'étude de la fréquentation des bars de nuit ${ }^{54}$ ), les régulations politiques (à travers l'étude de la politique municipale en matière de vie festive nocturne ${ }^{55}$ ), les régulations professionnelles (via une sociologie des patrons de bar ${ }^{56}$ ) et les régulations policières (via l'analyse de l'intervention des polices nationale et municipale la nuit).

D’autres entrées ont aussi été envisagées, comme celle de l'éclairage. Les «plans lumière » et autres «Schémas Directeurs d'Aménagement Lumière » ont déjà été interrogés par les urbanistes et les géographes qui s’intéressent aux évolutions de l'aménagement urbain ${ }^{57}$. D'autres auteurs, comme les membres du collectif Renoir ${ }^{58}$, se focalisent sur la valorisation des « ressources environnementales nocturnes » (paysages nocturnes, ciel étoilé, aurores boréales), la lumière (artificielle) étant alors plutôt considérée comme une pollution, ce qu'illustre le label «Commune pour l'amélioration de la qualité de la nuit ». Selon les contextes (urbains ou ruraux) et les priorités des pouvoirs publics, l'obscurité nocturne peut ainsi apparaître comme une contrainte (pour le maintien de l'ordre public ou le développement économique), qu'il faut lever par des politiques d'éclairage, ou bien comme une ressource à valoriser dans

52. La nuit a d'ailleurs ses experts qui interviennent régulièrement dans des conférences, colloques ou rapports sur le sujet, chose inimaginable à propos du jour ! Voir par exemple, en France, les travaux de Luc Gwiazdzinski : La Ville 24 beures sur 24. Regards croisés sur la société en continu, Paris, Éditions de l'Aube, 2003 ; La nuit, dernière frontière de la ville, Paris, Éditions de l'Aube, 2005 ; ou encore Nuits d'Europe. Pour des villes accessibles et hospitalières, Belfort, UTBM, 2007.

53. Voir encadré ${ }^{\circ} 1$ supra.

54. Voir encadré $\mathrm{n}^{\circ} 3$ infra.

55. Voir encadré $n^{\circ} 2$ infra. Pour une analyse plus dense des matériaux recueillis sur la politique municipale de régulation de la vie festive nocturne à Lille, voir CANDELA, "Concentrer la vie nocturne pour mieux la contrôler ? ", art. cité.

56. «Les patron.ne.s d'établissement de nuit entre production du désordre et gestion de l'ordre », communication dans la section thématique «L'action publique comme action collective : enquêter sur les acteurs "relais" de la régulation politique ", 14e Congrès de l'Association française de science politique, Montpellier, 10 juillet 2017.

57. Deleuil J.-M., Toussaint J. Y., « De la sécurité à la publicité, l'art d'éclairer la ville », Les Annales de la Recherche Urbaine, $\mathrm{n}^{\circ} 87,2000$, pp. 52-58 ; Fiori S., «Réinvestir l'espace nocturne, Les concepteurs lumière ", Les Annales de la Recherche Urbaine, n 87, 2000, pp. 7380 ; Challéat S., "Sauver la nuit ». Empreinte lumineuse, urbanisme et gouvernance des territoires, Thèse de doctorat de géographie, Université de Bourgogne, 2010 ; Mallet S., « Paysagelumière et environnement urbain nocturne », Espaces et Sociétés, n 14, 2011, pp. 35-52.

58. Challéat S., Lapostolle D., Benos R., "Consider the darkness. From an environmental and sociotechnical controversy to innovation in urban lighting », Articulo. Journal of Urban Research, n¹1, 2015 [http://articulo.revues.org/3064]. 
une démarche d'attractivité touristique. Ces considérations montrent en tout cas qu'en tant qu'objet d'action publique, la nuit peut être envisagée comme temps de repos ou temps de fête, comme contrainte à gérer ou ressource à développer. Les régulations politiques de la nuit s'avèrent donc multidimensionnelles et leurs orientations sont variables dans le temps et dans l'espace ${ }^{59}$.

C'est aussi cette question du gouvernement de la nuit qu'explore ce numéro de Cultures $\mathcal{E}$ Conflits, en particulier les contributions d'Étienne Walker et de Patrick Bruneteaux. S’appuyant sur le cas de Rennes, le premier met au jour différentes formes de gouvernementalité nocturne, impliquant autorités étatiques (préfecture), autorités municipales, professionnels et usagers de la nuit. Son travail s'inscrit dans un ensemble de travaux monographiques sur les "politiques de vie nocturne ", menés à Lyon, Nantes, Rennes ou Lille 60 mais aussi dans différentes villes anglaises ${ }^{61}$ ou encore néerlandaises ${ }^{62}$. Le travail de Patrick Bruneteaux s’intéresse pour sa part à un dispositif d'intervention sociale particulier : les maraudes de nuit auprès des personnes qui vivent dans la rue.

Certes, ces deux enquêtes analysent des politiques publiques dont les dynamiques d'élaboration sont le produit de jeux institutionnels et de logiques structurelles qui ne singularisent pas la vie nocturne. Examiner les politiques municipales de la nuit revient ainsi à montrer comment représentants locaux de l'État, élus locaux et agents publics territoriaux se positionnent les uns par rapport aux autres et définissent des champs d'intervention spécifiques (voir encadré $n^{\circ} 3$ ). Ces politiques constituent aussi des cas exemplaires de délégation des pouvoirs publics au secteur associatif. Quant aux maraudes nocturnes, elles soulignent l'ambivalence du travail social, qui apprend à endurer des conditions de vie difficiles plus qu'il ne peut proposer des solutions pour (s')en sortir. Pour Patrick Bruneteaux, l'étude de ce dispositif spécifiquement nocturne ne fait finalement qu'illustrer les contradictions de l’État social.

59. Contrastant avec la dénonciation de la « pollution » lumineuse, le projet de construction d'un phare à Paris dans le cadre de l'exposition universelle de 1889 illustre l'utopie d'une « abolition de la nuit » en lien avec le progrès technique. La «Colonne-Soleil » imaginée par l'architecte Bourdais et l'ingénieur Sebillot, d'une hauteur de 360 mètres, aurait permis d'éclairer un cercle de 11 kilomètres de diamètre et de consacrer définitivement Paris comme capitale des Lumières (Delattre S., Les douze heures noires, op. cit., pp. 168-172).

60. Deleuil J.-M., Lyon la nuit. Lieux, pratiques et images, Lyon, Presses Universitaires de Lyon, 1994 ; Beauparlant C., Darris G., Lemoine A. et Léon H., « La ville, la nuit. Rennes et Nantes, de nouvelles exigences de gestion urbaine ", Les cabiers de la sécurité, n 61, 2e trimestre 2006, pp. 85-107.

61. Hobbs D., Hadfield P., Lister S. et Winlow S., Bouncers. Violence and Governance in the Night-time Economy, Oxford, Oxford University Press, 2003 ; Hadfield P., Bar Wars, op. cit. ; Talbot D., Regulating the Night, op. cit.

62. Van Liempt I., Van Aalst I., "Urban Surveillance and the Struggle between Safe and Exciting Nightlife Districts ", Surveillance E Society, vol. 9, n³, 2012, pp. 280-292. Voir aussi, la présentation de différentes monographies dans Hadfield P. (ed.), Nightlife and Crime. Social Order and Governance in International Perspective, Oxford, Oxford University Press, 2009. 
En même temps, ces travaux dessinent aussi les singularités de la régulation ou du gouvernement de ce temps social particulier qu'est la nuit. D'une part, ils révèlent certaines activités qui s'y déroulent et qui n'ont pas lieu le jour ou pas avec la même intensité : les sorties en "boîte ", la consommation ostentatoire ${ }^{63}$, le binge drinking ou, plus largement, la consommation d'alcool en grande quantité, notamment chez les jeunes adultes ${ }^{64}$. Ils montrent bien, d'autre part, à quel point certaines pratiques (sortir en groupe, faire du bruit, crier...) sont construites comme des problèmes spécifiques à la nuit tant cette dernière demeure avant tout définie comme le temps du sommeil et du repos. Ils soulignent enfin l'isolement accru que produit le ralentissement de tout un ensemble d'activités pendant la nuit. C'est finalement dans cette tension entre temps d'activités «extraordinaires » et mise au repos des activités «ordinaires» (du moins les plus visibles d'entre elles) que se situe le temps de la nuit. C'est ainsi que les pouvoirs publics se trouvent investis du rôle d'arbitre entre des intérêts (ceux des riverains, commerçants, employés, usagers...) différemment affectés par les activités et les lieux de sociabilité nocturne. Mais les autorités sont aussi interpellées sur les services à mettre en place dans un temps de moindre présence sociale. Si la visibilité du «problème public » des sous-prolétaires à la rue dépend beaucoup de la saison (l'enjeu étant surtout porté médiatiquement en hiver du fait des conditions climatiques plus rudes), la visibilité plus grande des SDF la nuit appelle aussi des interventions spécifiques : alors qu'être SDF le jour c'est pouvoir se noyer dans la masse, être SDF la nuit c'est, au contraire, se retrouver (presque) seul.

\section{Encadré ${ }^{\circ} 2$ :}

\section{La fabrique diurne des politiques de la nuit à Lille}

Le collectif CANDELA s'est constitué alors que la vie nocturne devenait un enjeu saillant dans les débats politiques lillois. Entre 2010 et 2012, différents faits divers (fusillade à l'entrée d'une discothèque, décès - dans des circonstances encore troubles - de jeunes retrouvés noyés dans un canal, etc.) ont bénéficié d'un fort écho médiatique et ont été utilisés par les groupes d'opposition pour disqualifier l'action de la municipalité. C'est dans ce contexte que le collectif a entrepris, à la fin de l'année 2012, d'étudier les " politiques de la nuit » à l'échelle municipale. Il s'agissait d'étudier l'institutionnalisation et les transformations d'une politique qui repose sur des réglementations spécifiques (en particulier les horaires d'ouverture des bars, discothèques et épiceries de nuit), sur l'affectation de moyens spécifiques à la nuit (police municipale de nuit) ainsi que, jusque récemment, sur une sectorisation particulière de la ville (qui ne recouvre pas l'organisation en quartiers) définissant une "zone festive nocturne».

63. Voir Desjeux D., Charvin M., Taponier S. (dir.), Regards anthropologiques sur les bars de nuit. Espaces et sociabilités, Paris, L'Harmattan, 1999 ; Pinçon M., Pinçon-Charlot M., « Les nuits de Paris ", Les Annales de la Recherche Urbaine, n 87, 2000, pp. 15-24.

64. Massé B., "Rites scolaires et rites festifs : les "manières de boire” dans les grandes écoles », Sociétés contemporaines, $\mathrm{n}^{\circ}$ 47, 2002, pp. 101-129. 
Alors qu'étaient engagées, de façon exploratoire, les premières investiga-

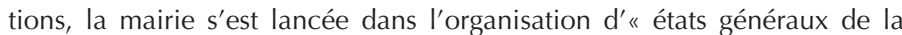
nuit » afin de réunir les protagonistes de la vie nocturne (patrons d'établissements de nuit, portiers, représentants des services de police, membres d'association de riverains, responsables de la vie étudiante dans les Universités et les écoles de la ville, services préfectoraux et municipaux, associations de prévention de l'alcoolisme, etc.) et de faire évoluer la réglementation. Armés du double statut de chercheur.e.s (a priori impartiaux) et d'universitaires en science politique (a priori rigoureux), les membres du collectif sont parvenus à négocier une position d'observateurs en échange de la rédaction des synthèses des tables rondes.

Cette entrée "sur le terrain » a livré un premier paradoxe : I'enquête sur la nuit se faisait essentiellement de jour! Si la recherche s'est accompagnée d'observations nocturnes dans le principal quartier festif de Lille, l'essentiel de l'investigation (entretiens, observations - notamment celles des Commissions de la vie nocturne qui ont lieu tous les deux mois -, archives administratives) a supposé une disponibilité diurne.

Cette enquête sur la fabrique de la politique de la nuit à Lille s'est ainsi avérée largement comparable à celles menées sur d'autres politiques publiques. Elle a par ailleurs mis en évidence des formes d'interactions observées dans d'autres espaces de construction de l'action publique. Les relations mairie/préfecture sont ainsi apparues très marquées par le juridisme du représentant de l' État dans les débats sur la « sectorisation ». Les patrons de bar présents aux États Généraux au titre de leurs responsabilités syndicales ou associatives se sont pour leur part montrés très soucieux de préserver leur monopole de la représentation de « la nuit », l'un d'entre eux dénonçant par exemple "l'intrusion » du patron d'un bar à shisha 65 .

Enfin, sans grande surprise, l'entre-soi de la Commission de la vie nocturne est d'autant plus sanctuarisé que les représentants de la profession ont été cooptés parmi les entrepreneurs les mieux établis. Si l'un d'entre eux est considéré par la presse locale comme le « pape de la nuit » du fait de la réputation et du succès de ses nombreuses boîtes de nuit, les autres patrons d'établissement disposent également de capitaux économiques et sociaux importants (souvent propriétaires de plusieurs enseignes lucratives - l'un d'eux a d'ailleurs développé un système de franchises - ils ont parfois diversifié leurs activités dans la restauration ou l'immobilier). Les capitaux et ressources qui comptent pour réguler la nuit ne sont guère originaux. Ce groupe d'établis peut ainsi œuvrer à la définition des normes et bons comportements professionnels comme lors des États Généraux, ou à l'occasion des commissions de la vie nocturne, où les gérants d'établissements légitiment leur activité autour de la figure de patron de bar " responsable », en distinguant leurs propres pratiques de celles d'autres établissements moins « recommandables».

65. Alors que celui-ci se plaignait de ne pouvoir se faire entendre et de ne pas avoir été invité aux États Généraux, le représentant syndical le rabroue : «Tu vois Tony, c'est pour ça que je t’ai demandé de te syndiquer! Si t'étais syndiqué, tu serais venu avec moi !» (Notes de terrain, 2e réunion du groupe de travail n ${ }^{\circ} 3$ des EGN, mairie de Lille, 15 mai 2013). 


\section{Quand la nuit bouscule (provisoirement) l'ordre social}

Les contours de la nuit peuvent aussi être dessinés en scrutant le(s) sens spécifique(s) dont elle est investie par des groupes sociaux particuliers. C'est alors toujours l'ordre social qui est au cœur des interrogations, mais la démarche consiste cette fois à examiner comment cet ordre est, si ce n'est vraiment remis en cause, du moins questionné ou légèrement bousculé par certaines pratiques nocturnes. Dans son travail sur les jeunes femmes dakaroises des quartiers populaires qui fréquentent chaque soir les lieux de sortie du centre de la capitale sénégalaise, Thomas Fouquet voit ainsi dans la nuit un « espace potentiel » au sens de Donald W. Winnicott, c'est-à-dire un espace (temporel ici) qui offre «la possibilité au sujet de mettre en cohérence certaines aspirations avec le monde social, matériel, environnant ». La fréquentation nocturne des bars et discothèques les plus en vue ne change pas la situation de ces jeunes femmes qui, chaque jour, retournent dans leur quartier. La nuit offre toutefois la possibilité d'une fiction de mobilité ascendante, qui permet un « voyage immobile », faute de pouvoir faire plus.

Au-delà du cas de ces jeunes femmes sénégalaises, les trois contributions qui portent sur la nuit festive (T. Fouquet, E. Walker, A. Zaytseva) livrent une image ambivalente des établissements " récréatifs » nocturnes : à la fois espaces marchands et espaces de sociabilité, espaces privés et espaces publics, espaces relativement autonomes par rapport aux autorités et espaces d'une coproduction du maintien de l'ordre fondée sur la « responsabilité sociale » des tenanciers, garants de la «bonne » alcoolisation ${ }^{66}$... Alors que, dans les représentations, la nuit festive apparaît souvent comme l'espace des transgressions, de la mise à distance des rôles (professionnels notamment) et de la remise en cause de l'ordre social ${ }^{67}$, on y constate une sélectivité sociale et spatiale des pratiques nocturnes, également mise en évidence dans l'enquête de CANDELA sur la fréquentation des bars lillois (voir l'encadré n³). Plus généralement, la substitution de certains codes vestimentaires (le costume professionnel abandonné au profit de tenues « de loisir »), la quête de l'amusement (et son potentiel carnavalesque) et l'usage de produits susceptibles de troubler la conscience de soi et des autres constituent a priori autant d'éléments permettant d'ériger la nuit en moment de bascule de l'ordre social, de son système de places et de prescription de rôles. Reste que l'interprétation de ces phénomènes, dont il ne faut par ailleurs pas exagérer la portée, demeure malaisée : outre qu'ils n'effa-

66. Ray Oldenburg qualifie d'ailleurs les bars de « tiers lieux » pour les distinguer des espaces domestique et professionnel (The Great Good Place: Cafes, Coffee Shops, Bookstores, Bars, Hair Salons, and Other Hangouts at the Heart of a Community, New York, Marlowe \& Cie, 1989).

67. Ainsi, dans la critique du récent film d'Edouard Baer (Ouvert la Nuit) parue dans Télérama ( $\mathrm{n}^{\circ}$ 3496, p. 58), l’errance du « héros » dans différents bars nocturnes parisiens est présentée comme illustrant « la capacité qu'a la nuit d'abolir les frontières entre les gens et les classes ». Cette vision de la nuit est aussi, en partie, celle véhiculée par le philosophe Michaël Foessel (La nuit, vivre sans témoins, Paris, Autrement, 2017). 
cent jamais les hiérarchies sociales sinon en instaurant leurs propres principes d'asymétries (qui a le droit d'entrer dans les établissements ? qui suscite le respect ou l'admiration la nuit ?, etc.), ils peuvent être lus comme autant de simulacres qui s'estompent à la levée du jour. L'interrogation est alors de nature ontologique : quelle est la « réalité », au sens ici d'objectivité sociologique, des rapports sociaux qui peuvent être observés au cours des espaces festifs nocturnes?

La sélectivité sociale est assurée par les logiques marchandes de solvabilité des usagers ou de détermination des critères de la «bonne » clientèle 68. Mais elle peut aussi être promue par les pouvoirs publics eux-mêmes, à travers les catégories servant à classifier la dangerosité potentielle des usagers de la nuit (étudiants vs. "jeunes de Lille Sud » dans le cas des administrations lilloises observées par CANDELA) ou à travers l'organisation de soirées destinées à des publics définis a priori comme non déviants, " responsables ", " autodisciplinés ». Cette dernière logique est très bien illustrée par la ville de Rennes et les « Nuits des 4 Jeudis » qu'elle organise (E. Walker) ${ }^{69}$.

Le cas des « clubs » de Saint-Petersbourg étudiés par Anna Zaytseva dans ce volume est encore plus saisissant : les cafés-concerts de la Russie postsoviétique, loin d'être des espaces de transgression des barrières sociales, sont avant tout des lieux où se pratiquent un « entre-soi élargi » et une économie affinitaire. C'est tout un travail d'entretien de l'ordre qui est à l'œuvre dans l'organisation des pratiques festives au sein des clubs. L'étude de la "fête ", comme objet et comme concept, donne ainsi à voir un contraste édifiant entre, d'une part, ce qui est vécu par les participants et par les pouvoirs publics sur le registre du désordre, de la transgression ou, plus simplement, des écarts aux rôles sociaux et professionnels investis le jour et, d'autre part, des pratiques largement organisées, institutionnalisées, régulées, ritualisées ou marchandisées.

Malgré les représentations dont elle est investie et l'opposition incluse dans l'expression "c'est le jour et la nuit », la nuit apparaît donc étroitement imbriquée au jour et l'ordre social qui s'y déploie y est très largement semblable, même s'il s'exprime à travers d'autres modalités. Si elle le remet en cause, c'est très provisoirement, comme pour les jeunes dakaroises des quartiers populaires. Pour autant, la nuit demeure historiquement le temps des « couvre-feux » en raison du potentiel de subversion que les pouvoirs publics lui assignent et de l'apparent brouillage des frontières entre espace public et espace privé. Il est significatif que l'ensemble des recherches menées par le col-

68. Voir notamment Marlière E., «Les vertus libératrices de la fête. Violences ritualisées et compétitions masculines ", Agora Débats/Jeunesse, n 53, 2009, pp. 35-48.

69. Voir aussi, à partir de l'exemple des « Nuits blanches », Evans G., « Hold back the night: Nuit Blanche and all-night events in capital cities ", Current Issues in Tourism, vol. 15, n 1-2, 2012, pp. 35-49. 
lectif CANDELA et toutes les communications proposées lors de l'atelier à l'origine de ce numéro thématique portent sur les espaces publics (rue, transports) ou ouverts au public (bars, clubs, boîtes de nuit, halls d'hôtel). La nuit étant aujourd'hui considérée comme l'espace-temps de la sphère privée la plus intime (de la chambre à coucher aux rêves qui peuplent le sommeil), celles et ceux qui y arpentent l'espace public incarnent, d'une manière ou d'une autre, une déviance : sans-abris qui reconstruisent un espace privé dans l'espace public (P. Bruneteaux) ${ }^{70}$; "aventurières de la nuit », fêtardes ou prostituées qui transgressent l'assignation des femmes à l'espace privé (T. Fouquet); jeunes qui s'enivrent «plus que de raison » dans l'espace public (E. Walker); concierges de nuit qui projettent un ensemble de représentations sulfureuses (T. Menoux) ${ }^{71}$. Les propos de la préfète de Bretagne, rapportés par Etienne Walker, illustrent bien la façon dont la «nuit publique » peut engendrer des fantasmes de transgression: «C'est hallucinant. Vous avez là des gens tout à fait normaux. Pas des SDF ni des marginaux, même s'il y en a quelques-uns. Non, des gens normaux, étudiants des écoles d'ingénieurs, en maîtrise ou en doctorat [...] Ces gens ingurgitent tout cela, se déshabillent, font partout... Il y a des accouplements en plein air, des hurlements ». La nuit apparaît, aux yeux de celle qui incarne les pouvoirs publics, comme le moment où la normalité se mue en déviance ${ }^{72}$ et lui fournit l'occasion de rappeler publiquement certaines des frontières qui régissent et distinguent théoriquement espaces publics et privés (nudité, relations sexuelles, consommation d'alcool...).

\section{Encadré $\mathbf{n}^{\circ} \mathbf{3}$ : Sociologie des publics des bars lillois}

Une enquête par questionnaire a été réalisée en 2014 (un mercredi et un jeudi) et en 2015 (un samedi), dans le cadre du cours de " méthodes des sciences sociales " de la licence de science politique de l'Université de Lille. 1054 clients de bars lillois ouverts après 20h ont été interrogés. L'enquête met en évidence que les bars du secteur de la vie nocturne sont majoritairement fréquentés par une population jeune (seuls 6,1\% ont plus de 45 ans), diplômée (60\% ont un diplôme de l'enseignement supérieur long) et, dans une moindre disproportion, masculine $(55 \%$ d'hommes pour $45 \%$ de femmes). Tandis que les classes moyennes et supérieures sont surreprésentées $(21,4 \%$ des interviewés déclarent avoir des parents « cadres et professions intellectuelles »), cette population se caractérise par la richesse et la diversité de ses pratiques culturelles (ils se rendent trois fois plus au concert classique et plus de quatre fois plus au concert jazz que la moyenne des Français). La fréquentation du bar la nuit en semaine participe du style de vie propre aux jeunes dotés en ressources économiques et culturelles. On a donc bien affaire à un usage socialement situé de ces espaces publics nocturnes.

70. Voir aussi Gaboriau P., Clochard. L'univers d'un groupe de sans-abriparisiens, Paris, Julliard, 1993.

71. On pourrait d'ailleurs suggérer que des quidams qui ne feraient que se balader la nuit pourraient paraître suspects ou déviants aux yeux des usagers de la nuit précédemment cités. 
Si les clubs nocturnes de Saint-Pétersbourg étudiés par Anna Zaytseva échappent à ce type de jugement, c'est qu'ils recréent un entre-soi domestique, un espace pour des proches, dans des lieux a priori publics. Le « relâchement » s'accompagne alors d'un "contrôle mutuel », si bien que "l'ordre négocié de la nuit » échappe au contrôle public. Là où les pratiques festives se déroulent dans des espaces dérobés, «fermés », les règles s'articulent autour de l'imaginaire de la «maison », sous le régime de la domesticité, de la familiarité, d'un entre-soi construit, comme dans ces cafés pétersbourgeois. À l'inverse, là où la « fête » déborde dans l'espace public, devenant visible (et audible !), l'ordre public troublé se rappelle aux participants par l'encadrement, le contrôle et la discipline. La nuit pose ici de façon cruciale la question de la cohabitation des publics et des mécanismes de régulation sociale et politique qui l'encadrent.

\section{Nuit subie, nuit désirée : les appréhensions de la nuit comme principe de classement}

Au regard des recherches ici réunies et de leur confrontation, force est d'admettre que l'on peut travailler sur la nuit sans en offrir une définition figée, en la définissant simplement comme une fraction du nycthémère qui n'est pas le temps ordinaire des activités sociales (« le jour»). Car, suivant les articles, la nuit dont il est question est tantôt celle que définissent les pouvoirs publics (État, municipalités), à travers un ensemble de réglementations ou dispositifs applicables à certains horaires (et relatifs au bruit, à l'occupation de l'espace public, aux activités de commerce...), tantôt celle que définit une profession et ceux qui la régulent, ou le temps de pratiques (notamment festives) particulières. Les pouvoirs publics circonscrivent une séquence temporelle nocturne pour en faire un espace-temps à réguler, à encadrer, à contrôler, voire à réprimer, mais aussi à organiser, en raison des logiques bureaucratiques d'organisation des services et des équipes et de détermination des horaires de travail des agents. Les définitions institutionnelles de la nuit et de ses frontières constituent par ailleurs un enjeu fondamental du point de vue des activités salariées et de leur rémunération, en témoignent les récentes révisions des qualifications juridiques du travail de nuit en France. En effet, les lois Macron et El Khomri ont non seulement légalisé l'extension des horaires

72. L'enquête menée par CANDELA sur la régulation de la vie nocturne lilloise, notamment à l'occasion des États Généraux de la Nuit, montre cependant à quel point les catégorisations ordinaires du monde social demeurent partagées par la plupart des acteurs qui participent à la production du cadre réglementaire local, qu'ils soient élus, policiers, fonctionnaires de la mairie ou représentants des patrons d'établissement. Tandis que les jeunes des quartiers populaires voient leurs pratiques délinquantes naturalisées ( les prédateurs de la nuit ») car rapportées à leur identité de "racailles », les déviances des « étudiant.e.s » (i.e. celles et ceux définis comme client.e.s « légitimes » des établissements) leur sont rarement imputées mais attribuées à une pluralité de causes qui, dès lors, minimisent leur responsabilité : alcool, insuffisant contrôle des établissements universitaires sur les associations étudiantes, "société » qui se détraquerait, délitement des liens familiaux, etc. Dans les discours des acteurs publics, contrôler, interpeller et parfois sanctionner ces étudiant.e.s viseraient avant tout à les protéger. 
d'ouverture des commerces mais ont surtout réduit les horaires considérés comme relevant du travail de nuit (auparavant fixés, sans condition, de $21 \mathrm{~h}$ à 6h) ${ }^{73}$. Catégorie d'action publique, la nuit ou plutôt la vie nocturne peut donc être appréhendée comme un « concept sténographique », pour parler comme Jean-Claude Passeron ${ }^{74}$, c'est-à-dire une appellation institutionnelle, un objet socialement préconstruit qui charrie un ensemble de prénotions en matière d'interventions des pouvoirs publics.

Pour un collectif de travail comme celui d'un hôtel, la nuit est aussi une séquence temporelle, qui amène une organisation du travail particulière, $\mathrm{du}$ fait de la présence d'un personnel moins nombreux ou du déploiement d'activités spécifiques. Quant aux pratiquants de la nuit festive, les limites de leur nuit sont beaucoup plus indéterminées. Les nuits des uns et des autres ne se superposent pas mais elles ne sont pas sans lien : elles se chevauchent en partie et, par ailleurs, elles se définissent toutes par une forme de régime d'exceptionnalité temporaire à l'échelle des cycles de 24 heures.

La délocalisation et la transnationalisation des activités économiques ont aussi donné naissance à des temporalités subalternes mondialisées inédites, à l'instar des centres d'appel à Manille, étudiés par Jérôme Tadié, organisés autour des horaires états-uniens (de 21 heures à 6 heures du matin pour ceux qui traitent avec la côte Est, de 1 heure à 10 heures du matin pour ceux qui travaillent avec la côte Ouest). Non seulement cette nuit américaine ainsi étendue contribue à brouiller davantage la frontière diurne-nocturne, mais ces nouveaux archipels nocturnes (calqués sur les quartiers d'affaires américains diurnes, avec leurs fast-foods, chaînes de café internationaux et autres supérettes permettant de déjeuner vers 2 heures du matin), se sont implantés dans la capitale philippine en complète déconnection avec les espaces noctambules de la ville... amenant aussi une transformation des rythmes liés au divertissement : les heures de pointe des bars se situent désormais autour de 6 heures du matin 75 .

Cette évolution des conceptions de la nuit et de la vie nocturne légitime rappelle à quel point le temps est non seulement une construction sociohistorique, mais aussi une «institution sociale contraignante 76 ». Il n’y a pas pour autant homogénéisation des perceptions de la nuit. En tant que dimension du

73. Selon la loi Macron, dans les commerces de vente en détail situés dans les «zones touristiques internationales ", le début du travail de nuit peut être reporté jusqu’à minuit. La Loi El Khomri modifie, quant à elle, la définition même du «travail de nuit ». Ainsi, des salariés qui débutent leur journée de travail à 5 h ne voient plus leurs heures faites entre 5 h et 6 h considérées comme travail de nuit.

74. Passeron J-C., Le raisonnement sociologique. L'Espace non-poppérien du raisonnement naturel, Paris, Nathan, 2006, p. 114.

75. Tadié J., "Nuits américaines à Manille. Centres d'appels et nouveaux quartiers nocturnes », Sociétés politiques comparées, n³8, janvier-avril. 2016 [http://www.fasopo.org/ sites/default/files/charivaria3_n38.pdf].

76. Elias N., Du Temps, Paris, Fayard, 1996. 
rapport au temps, le rapport à la nuit demeure différencié selon les groupes sociaux et reste le produit de socialisations plurielles ${ }^{77}$. Pour les SDF comme pour les concierges de nuit d'hôtel de luxe, étudiés respectivement par P. Bruneteaux et T. Menoux, la nuit est très largement vécue sous l'angle de la contrainte. Inversement, les noctambules de Rennes, de St-Pétersbourg ou de Lille entretiennent un rapport hédoniste à la nuit. Recherchée, elle permet l'entretien de réseaux de sociabilité mais permet aussi de cultiver un entre-soi confortable. Il y a là une forme de distinction chez les happy few de la vie nocturne, qui peuvent consommer de manière ostentatoire (y compris le temps). Pour les " aventurières de la nuit », enfin, le rapport au temps est sans doute plus ambigu. Si la nuit est désirée en ce qu'elle offre l'opportunité de valoriser leur capital esthétique, l'analyse ne peut occulter à quel point cette appréhension positive de la vie nocturne est largement structurée par des rapports de domination locaux et internationaux qui encadrent leurs possibles.

Aussi, prise dans cette tension entre l'emic (la perspective des observés) et l'etic (la perspective de l'observateur), la nuit nous apparaît comme une entrée heuristique pour les sciences sociales du politique. Étudier le monde social à travers le rapport à la nuit, c'est la considérer à la fois comme un enjeu de luttes et comme un espace où s'expriment les rapports sociaux (de genre, de classe, de racialisation, de sexualité ou de génération). Ce numéro a pour ambition de démontrer la pertinence de ce champ de recherche en constitution en pointant du doigt les nombreuses zones de turbulences qu'il révèle.

77. Sur le rapport au temps comme « disposition» acquise dans les processus de socialisation primaire et secondaire, voir Darmon M., Classes préparatoires. La fabrique d'une jeunesse dominante, Paris, La Découverte, 2013, p. 186. 